\title{
EL "CCHACCO" : COMPOSICION FISICO-QUIMICA Y USO ALIMENTARIO
}

Dániza M. Guerrero Alva

\section{RESUMEN}

La gran diversidad de recursos alimenticios que ofrece nuestro país, no sólo tiene origen agrícola o pecuario, sino también mineral. Este es el caso del "cchacco», arcilla comestible, considerado como suplemento dietético de gran consumo entre los pobladores del altiplano peruano-boliviano. El objetivo del estudio fue el de conocer más sobre las características físicas y químicas de este producto, establecer la calidad sanitaria del mismo, así como formular una mezcla que incluyera " cchacco".

Los análisis físicos y químicos fueron desarrollados de acuerdo a los métodos aplicables a alimentos. Los constituyentes analizados fueron : bumedad 7,78\%, sólidos totales $92,21 \%$, cenizas $91,80 \%$, nitrógeno $0,0028 \%$, proteína $0,0174 \%$, pH de 6 y densidad de carga $0,6936 \mathrm{gr} / \mathrm{ml}$. Adicionalmente se balló creatinina $3,58 \mathrm{mg} \%$, glucosa $225 \mathrm{mg} \%$ y restos de vegetales.

La calidad sanitaria del producto fue determinada, dadas las condiciones de manipuleo y expendio observadas. Los resultados fueron negativos para los siguientes microor

ganismos: enterobacterias, estreptococos, estafilococos, vibrium cólera, salmonella, bacilo tuberculoso, bongos y levaduras.

Finalmente, mediante la preparación de una mezcla tipo salsa, se definió la cantidad adecuada de " cchacco* que debe añadirse, así como la mejor alternativa de sabor.

\section{INTRODUCCION}

El " cchacco", ha sido definido por Ludovico Bertonio en 1,612 como una «tierra usada para fabricar ladrillos y que las mujeres aymaras consumían como alimento (1). Antúnez de Mayolo (2) considera a este silicato de alúmina o arcilla en estado.coloidal ("cchacco»), bajo el acápite de "suplemento mineral» como un aporte de la dieta pre-hispánica.

Las personas consumidoras de" cchacco" atribuyen numerosas bondades a este producto. Aseguran que cura las úlceras, coadyuva a la buena digestión de los alimentos, y bajo la forma de cataplasmas se le emplea para el dolor de cabeza y de muelas, siendo alimento obligado de mujeres gestanteș. No falta quienes aseguran que también es eficaz contra el cáncer.

Esta arcilla es ampliamente conocida en Puno, desde donde se comercializa a otros lugares del sur peruano, como Tacna, envasada en saquillos de tocuyo o yute.
La venta es al menudeo durante las ferias agropecuarias, sin observar las normas mínimas de higiene y cuidado, a pesar del uso alimentario al que se destina.

EI consumo se hace directamente o bajo la forma de salsa; es decir, disuelto en agua y sal para mejorar el sabor. Se sirve tradicionalmente con papas, (para acompañar las huatias) chuño, pero también con queso o camotes como lo hacen en Tacna.

El presente trabajo tiene por finalidad ampliar el conocimiento de las características físicas y químicas del" cchacco", evaluar su calidad sanitaria y desarrollar una formulación con buenas características sensoriales para consumidores no habituados al uso de arcillas en la dieta diaria.

\section{REVISION DE LITERATURA}

Este producto comestible ha sido estudiado tı cuanto a los elementos que lo constituyen con los siguientes resultados: 
Cuadro No. 1: 'componentes del "Cchacco"

\begin{tabular}{|l|c|}
\hline COMPONENTE & CANTIDAD \\
\hline Sílice & \\
Oligoelementos & \\
\hline Sílice & 54,40 \\
Alúmina & 23,40 \\
Peróxido de fierro & 6,90 \\
. Magnesio & 2,80 \\
. Agua, Potasa y Man- & 10,50 \\
ganeso & \\
\hline
\end{tabular}

Fuente :Antúnez de Mayolo (1988) y Frisancho (1990).

De acuerdo con estos reportes, la sílice presente actuaría como eficaz desinflamante y los oligoelementos le confieren características específicas, relacionadas con : tamaño de partícula, área superficial, grado de solubilidad y grado de asimilación.

Los elementos hallados: fierro, magnesio, potasio y manganeso son de notable importancia en la alimentación.

El hierro está presente en todas las células del organismo, jugando un rol importante en las reacciones bioquímicas, por formar parte de enzimas que transportan electrones (citocromos), de la activación del oxígeno (oxidasas y oxigenasas) y del transporte del oxígeno (hemoglobina y mioglobina) (3).

Su metabolismo posee dos circuitos: el primero interno, con reutilización continua de fierro a partir del catabolismo de los glóbulos rojos, y el segundo, repre sentado por las pérdidas de fierro del organismo, y la absorción de fierro ferroso a fierro férrico.

El balance de fierro se mantiene gracias a los siguientes mecanismos: a) reutilización continua del hierro de las células catabolizadas en el organismo; b) la existencia de ferritina o proteína de depósito; c) la regulación de la absorción de fierro afectada por requerimientos reales, con un aumento de la absorción en deficiencia y una disminución en sobrecarga. Finalmente, se conoce además, su importancia en la síntesis de colágeno y para la hidroxilación de la prolina y lisina en el procolágeno, constituyente de la matriz extracelular. Se le absorve con mayor facilidad de las carnes y vísceras; y, en presencia de ácido ascórbico, en menor cantidad, de los vegetales. Su asimilación puede inhibirse por los fitatos y taninos.

La carencia es más común en niños y madres en la etapa de gestación o amamantamiento, produciéndose anemia (4).
Con respecto al magnesio, éste se halla en los huesos (combinado con fosfatos y bicarbonatos), lugar de reserva para épocas de escasez.

Se le puede hallar en mayor concentración que cualquier otro mineral (salvo el potasio), en las células de los tejidos blandos, jugando un rol fundamental en la respiración celular (fosforilación oxidativa) que conduce a la formación de ATP, ADP y AMPc.

Trabaja como activador de todas las reaccionesenzimáticas que requieren tiamina pirofosfato (TPP) y de las reacciones del metabolismo de lípidos y proteínas, teniendo gran importancia en la transmisión y actividad neuromuscular, actuando en algunas etapas sinérgicamente con el calcio y, en otras, como antagonista de este elemento.

Se le halla en la clorofila, por ello su deficiencia dietaria es poco probable, afectando su exceso el aprovechamiento de calcio y fósforo.

Bajo la forma de óxido de magnesio se le emplea en la farmacopea como purgante.

Por otro lado, el potasio es el principal catión en el fluído intracelular y el cuerpo humano puede contener aproximadamente $2,6 \mathrm{gr}$. de potasio/kg de tejido libre de grasa. Tanto las células del músculo como de los nervios son ricas en potasio. Este ión es importante para el metabolismo de los carbohidratos y proteínas, pero su mecanismo de acción es aún desconocido. Ejerce acción complementaria a la del sodio en el funcionamiento de las células.

Los vegetales son ricos en potasio, de allí que una dieta en base a estos alimentos, puede producir exceso de potasio que se excreta tan fácilmente como se absorve, ya sea vía urinaria o por la transpiración, pero en enfermedades renales, diabetes o en cuadros de diarreas y vómitos, puede disminuir en el organismo. La deficiencia causa debilidad muscular y puede derivar en parálisis o paro cardiaco.

=Este elemento bajo la forma de hidrato de potasio o potasa, que reporta Chervin (5) en la composición química del " cchacco", es un veneno muy enérgico, por tanto es obvio que su existencia en el producto debe ser corroborada.

De igual manera, el manganeso se presenta en altas concentraciones en huesos, hígado y riñones, actuando como un cofactor enzimático y constituyente de metaloenzimas. El manganeso también está relacionado estrechamente con el metabolismo de los carbohidratos y lípidos, como también con la función cerebral (6). Otros elementos como el aluminio están siendo investigados para determinar su función e importancia dentro del organismo humano. 
.Para conocer algo más sobre las características químicas y físicas del " cchacco" se desarrolló determinaciones de humedad, sólidos totales, cenizas, nitrógeno, $\mathrm{pH}, \mathrm{y}$ densidad de carga en el producto en polvo. Adicionalmente se buscó la presencia de creatinina y glucosa en la muestra suspendida en agua destilada.

Dadas las condiciones tan precarias de comercialización, se deseó precisar la calidad sanitaria del producto y si podía producirse algún tipo de contaminación por mal manipuleo. Para ello se investigó la presencia de entero bacterias, estreptococos fecales, estafilococos, cocos, vibrium cólera, salmonella, bacilo tuberculoso y hongos (7).

Debido a las características del producto en polvo, se estimó conveniente su aplicación en la formulación de una salsa que contuviera además proteína láctea, almidón, vegetales deshidratados, saborizantes y agua, cantidades diferentes de "cchacco".

Estas muestras fueron analizadas por un panel de evaluación semi-entrenado, teniéndose especial cuidado en determinar la cantidad máxima del producto que fuera aceptado sin disminuir el grado de calidad de los atributos observados (8).

También se estableció la relación entre el sabor del " cchacco" con el supuesto ingrediente responsable de ese atributo.

Definida la mejor composición, se evaluó sensorialmente para hallar la alternativa de sabor ópti$\mathrm{ma}$, de acuerdo al siguiente esquema de trabajo :

\section{Formulación base

$$
\mathrm{M}, \mathrm{E}, \mathrm{X}
$$

\begin{tabular}{|c|}
\hline Ev. Sensorial \\
(prueba de ordenamiento) \\
donde: $M$--- 4,5 gr. de maní \\
E -- 9,0 gr. de nueces \\
X -- 13,5 gr. de ají
\end{tabular}

Finalmente, se determinó la composición química de la fórmula con el contenido óptimo de "cchacco", así como la de rnejor alternativa de sabor

\section{RESULTADOS Y DISCUSION}

\section{a). Análisis físico - químico de la muestra :}

Antes de efectuar los análisis, la muestra fue molida muy finamente para obtener mejores resultados.
Los valores de los constituyentes y los métodos (9) usados se citan a continuación:

CUADRO N ${ }^{\circ}$ 2: ANALISIS QUIMICO DEI CCHACCO"

\begin{tabular}{|c|c|c|c|}
\hline \multicolumn{2}{|l|}{ CONSTITUYENTE } & METODO & RESUITADO \\
\hline HUMEDAD & (\%) & Estufa & 7,78 \\
\hline SOLIDOS TOTALES & (\%) & Diferencia & 92,22 \\
\hline CENIZAS & (\%) & Calcinación & 91,80 \\
\hline NITROGENO & (\%) & $\begin{array}{l}\text { Semi micro } \\
\text { Kjeldhal }\end{array}$ & 0,0028 \\
\hline $\begin{array}{l}\text { PROTEINA } \\
\mathrm{pH}\end{array}$ & $(\%)$ & "Potenciómetro & $\begin{array}{l}0,0174 \\
6\end{array}$ \\
\hline $\begin{array}{l}\text { DENSIDAD DE CARG } \\
(\mathrm{gr} / \mathrm{ml})\end{array}$ & & $\begin{array}{l}\text { Producto en } \\
\text { polvo }\end{array}$ & 0,6936 \\
\hline
\end{tabular}

En el cuadro anterior se aprecia que el contenido de humedad es muy bajo y semejante a algunos alimentos andinos (como "cañihua" con $8,1 \%$ y maní $7 \%$ ), o productos industrializados muy concentrados 0 deshidratados (caramelo $7 \%$, curry en polvo $9 \%$ o pan de centeno tostado $9 \%$ ), (10).

El contenido de cenizas constituye, casi integramente, los sólidos totales del producto. Cabe resaltar que ningún alimento posee porcentaje tan elevado de cenizas: $7,9 \%$ para levadura de cerveza en polvo, $8,5 \%$ de cenizas (en base seca) para queso tipo "roquefort" de ocho semanas de maduración (11); ó $7,12 \%$ de ceniza para un alimento de consumo humano, preparado a partir de la mezcla de afrecho, úrea y melaza, se consideran altos y poco comunes (12).

Al analizar el contenido de proteína por el método semi-micro Kjeldhal, éste fue muy pequeño $(0,0174 \%)$, pudiendo corresponder a restos de plantas o animales.

La presencia de proteína fue corroborada por un segundo análisis efectuado por otro método, determinándose 1,27 gr\% de proteína plasmática en la muestra. El resultado obtenido por elprimer método es menor al de las frutas que por lo general presentan bajos porcentajes de proteína $(0,3 \%$ en manzanas, membriIlo, níspero y uva, o chicha de yuca con $0,1 \%),(13)$.

Respecto al $\mathrm{pH}$, el valor hallado fue de 6 y permite clasificar al "cchacco" como un alimento de baja acidez, compartiendo el mismo grupo con la leche, carnes y hortalizas (14).

Finalmente, la densidad de carga determinada de

$0,6936 \mathrm{gr} / \mathrm{ml}$, fue algo superior a la de la leche en polvo instantaneizada, producto de la eficiente molienda previa al análisis (15).

En la muestra se determinó también glucosa, hallán- 
dose $225 \mathrm{mg} \%$. A este compuesto se le llama «azúcar de uva", dextrosa o dextroglucosa y se le encuentra en los seres organizados, principalmente vegetales que, posiblemente, son la fuente que justifica su presencia en el "cchacco".

Constituye gran parte de la materia azucarada de las uvas, de la orina de los diabéticos y asociada a la levulosa, forma los principios azucarados de la mayor parte de los frutos ácidos y, también, se le encuentra en la miel, en el desdoblamiento del azúcar de caña y en polisacáridos, entre los que figuran la dextrina, féculas, celulosas y otros hidratos de carbono, además de los glucósidos.

La glucosa puede formarse siempre que se haga actuar sobre los compuestos citados, ácidos débiles o diluídos. La cantidad de glucosa contenida en los divirsos frutos es variable, dependiendo no sólo de las diferentes especies de frutos, sino de su grado de madurez. Así por ejemplo :

\begin{tabular}{|l|c|}
\hline Fruto & $\begin{array}{c}\text { Cant. por } 100 \text { grs. } \\
\text { de glucosa }\end{array}$ \\
\hline Melocotón & 1,50 \\
Albaricoque & 1,80 \\
Fresas & 5,70 \\
Cerezas & 8,70 \\
Uvas & 15,00 \\
\hline
\end{tabular}

Finalmente, en el producto se halló $3,58 \mathrm{mg} \%$ de creatinina. Esta sustancia, descubierta por Liebig, se forma por eliminación de agua de la creatina, al calentarse con ácidos concentrados. Es un producto de secreción en animales y el hombre, hallándosele en la orina y en el mísculo de los crustáceos, siendo los primeros muy posiblemente la fuente de origen de este compuesto en el "cchacco".

La creatinina se caracteriza por cristalizar en prismas clinorrómbicos. Da reacción alcalina y su sabor es cáustico. Por oxidación forma metilguanidina, al reemplazar de uno a dos átomos de hidrógeno con el radical hidroxiglicólico del ácido glicólico. También puede formarse a partir de la creatina, por ebullición prolongada en metio acuoso o en ácidos concentrados (ácido sulfúrico, fosfórico o nítrico) con eliminación de agua, de acuerdo a la siguiente reacción :
C $\mathrm{H} \mathrm{N}$
4932
C $\mathrm{H} \mathrm{N} \mathrm{O}$
H $O$
473
2

\section{CREATINA CREATININA}

Tanto la creatina como la fosfocreatina son compues- tos importantes en los mecanimos de almacenamiento ytransmisiónde moléculasfosfatadas, altasenenergía. En lossiguientes esquemas se puede observar la síntesis de este compuesto, a partir de los aminoácidos y su presencia en el catabolismo de aminoácidos excedentarios, debido al consumo excesivo de proteina en relación a las necesidades del individuo o cuando la distribución de aminoácidos de la proteína presenta un aminoácido limitante. En estos casos, los aminoácidos se metabolizan rápidamente; el esqueleto carbonado se emplea como fuente de energía y a su vez, el grupo amino puede serlo de creatinina en la orina (16).

\section{ESQUEMA DE LA SINTESIS DE CREATINNA A PARTIR DE AMINOACIDOS}

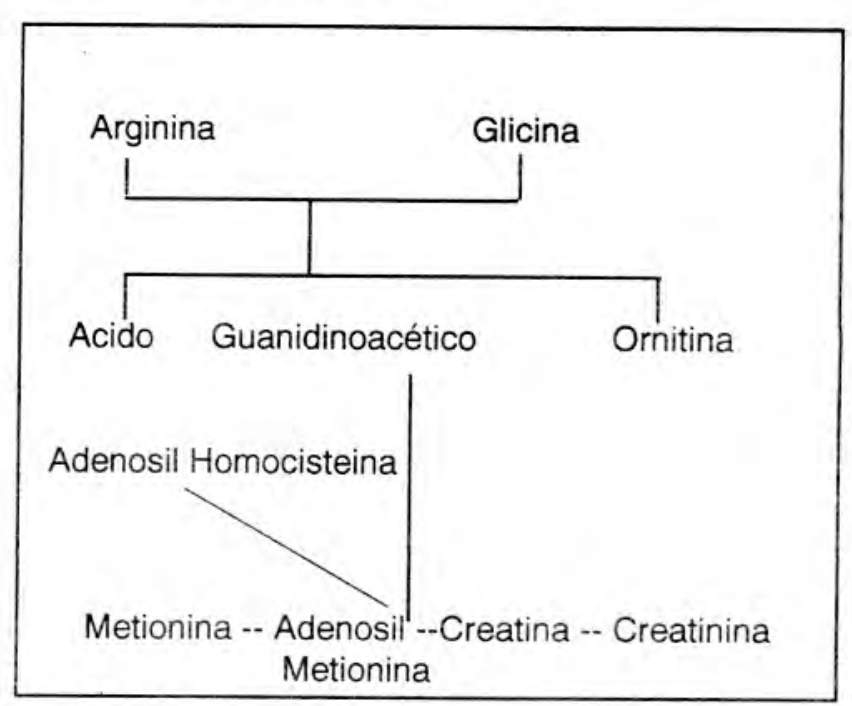

ESQUEMA DELCATABOLISMO EXCEDENTARIO DE AMINOACIDOS

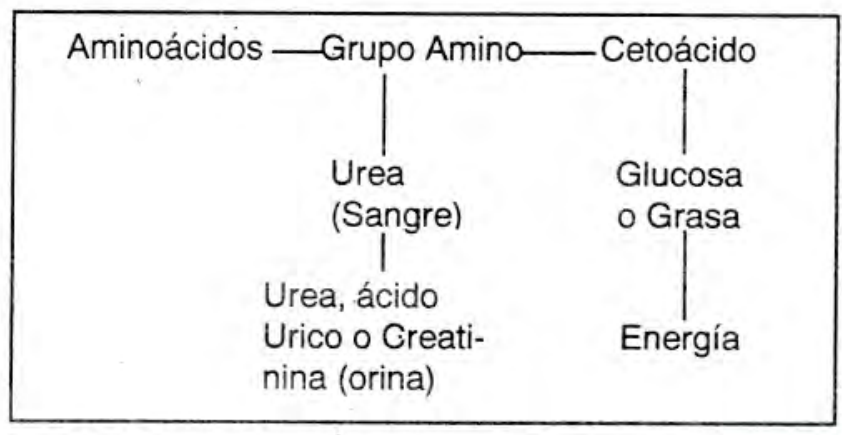

\section{b). Análisis Microbiológico}

Para los cultivos, la muestra fue previamente incubada en caldo triptrina soya por 24 horas. Las tres últi- 
mas pruebas citadas en el cuadro fueron hechas suspendiendo la muestra en agua destilada estéril. Los análisis microbiológicos tuvieron los siguientes resultados:

\section{Cuadro $\mathbf{N}^{\circ} 3$ ANALISIS MICROBIOLOGICO DEL CCHACCO}

\begin{tabular}{|l|l|c|}
\hline \multicolumn{1}{|c|}{ Medio } & Microorganismo & Resultado \\
\hline Agar sangre & $\begin{array}{l}\text { Streptococcus } \\
\text { y Staphylococcus }\end{array}$ & \\
Agar azida & Streptococcus & \\
Mackonkey & Bacterias & Negativo \\
Manitol & Cocos & en \\
TBS agar & Vibrio cholerae & todos \\
Agar Salmo- & Salmonella & los \\
nella & Enterobacterias & medios \\
Gram & Hongos (ex.Dir) & \\
KOH & Bacilo tubercu- & \\
BK & loso & \\
Sabourand & Hongos & \\
\hline
\end{tabular}

Estos resultados negativos indican que, a pesar de las condiciones antihigiénicas en las que se comercializa :

a) Es un producto natural que no es susceptible a sufrir contaminación microbiana presentando calidad sanitaria óptima y estable;

b) No se puede producir alteraciones o deterioro del producto por agentes microbianos;

c) No puede producir en las personas que lo consumen como alimento o fines medicinales, enfermedades de etiología microbiana transmitida por alimentos;

d) No necesita adición de conservadores;

e) También se pudo observar pequeños vegetales en una muestra de "cchacco".

d). Consumo de arcillas
Este consumo poco común se registra también en pobladores de algunas zonas de Oriente y Occidente. Sin embargo sus detractores la definen como " pica" o "malacia".

En el primer caso la voz proviene del latín pica, o sea "urraca», por la propensión de esta ave a comer cualquier cosa. Se considera una depravación del apetito, por desear substancias inusitadas o dañosas y no alimenticias (17). Un ejemplo típico es el consumo de dolomita, roca sedimentaria rica en calcio y magnesio, muy insoluble aún pulverizada e inadecuada, según se dice, para el consumo humano; siendo su uso una reminiscenla "pica" o apetito por arcilla o tiza observada durante la histeria o el embarazo (18).

La "pica» también pueden padecerla los niños de corta edad, y según los nutricionistas, se debe a la deficiencia en fierro. Las más usuales son:

. Ingesta de hielo .... pagofagia.

. Ingesta de tierra, lodo, arcilla...teofagia

que desaparece con el consumo de fierro.

Es posible que tanto la cultura como la tradición promuevan esta tendencia. En el sur de los Estados Unidos, se cree que el consumo de arcilla, almidón de maíz o sosa, calman las náuseas, cefáleas, mareos, vómitos y aseguran una prole sana y hermosa. Tambien la relacionan con factores psicológicos, especialmente en niños.

En Irán, se dice que la deficiencia en zinc promueve el consumo de arcillas, algunas de las cuales son ricas en hierro y zinc, (19).

Sin embargo, cada vez más crece el número de personas que se inclinan por la alimentación y curación de enfermedades en forma natural. Por tanto, no sólo podría ser importante esta arcilla como fuente de minerales, sino por la forma en la que están presentándose estos elementos que pudieran tener un comportamiento semejante a la fibra dietaria (soluble o insoluble). Estas fracciones que forman parte de los alimentos vegetales han tomado notable importancia en estas últimas décadas, no sólo porque permite el adecuado funcionamiento del estómago, sino por la relación que existe entre la ingesta de fibra y el cáncer alcolon. Esto podría justificar el porqué algunas personas aseguran que evita o cura el cáncer, y la baja incidencia de este tipo de neoplasia en Puno, dondelos pobladores se alimentan con cereales andinos como: kiwicha», "cañihua» o quinua (de $5,76 \%$, $12,92 \%$ y $5,31 \%$ respectivamente), "cchacco" y "fasa" (20).

\section{e). Aplicación tecnológica del «cchacco»}


De acuerdo a las características químicas y microbiológicas del producto, se desarrolló una formulación tipo salsa con diferentes cantidades de la muestra de acuerdo al siguiente cuadro:

\section{Cuadro $N^{\circ} 4$ : COMPOSICION DE LAS FORMULACIONES EN BASE A CCHACCO}

\begin{tabular}{|l|l|llll|}
\hline \multirow{2}{*}{$\begin{array}{l}\text { composicio } \\
\text { (gr) }\end{array}$} & \multicolumn{5}{|c|}{ FORMULA } \\
\cline { 2 - 6 } & Patrón & L & V & B & K \\
\hline $\begin{array}{l}\text { Proteína } \\
\text { láctea }\end{array}$ & 22,642 & 22,642 & 22,642 & 22,642 & 22,642 \\
$\begin{array}{l}\text { Carbohidra- } \\
\text { tos }\end{array}$ & 11,69 & 11,69 & 11,69 & 11,69 & 11,69 \\
$\begin{array}{l}\text { Vegetales } \\
\text { Deshidra- } \\
\text { tados }\end{array}$ & 0,16 & 0,16 & 0,16 & 0,16 & 0,16 \\
Sal & 0,5 & 0,5 & 0,5 & 0,5 & 0,5 \\
Agua & 50 & 50 & 50 & 60 & 70 \\
Cchacco & 00 & 1,0 & 2,0 & 3,0 & 4,0 \\
\hline
\end{tabular}

Mediante un test sensorial descriptivo aplicado a once evaluadores, se determinó la calidad final de cada fórmula y su relación con el contenido de muestra.

\section{RESULTADO DEL TEST DESCRIPTIVO}

\begin{tabular}{|l|llll|}
\hline & $\begin{array}{c}\text { Cantidad de } \\
\text { Cchacco }\end{array}$ \\
Calidad & & & & \\
Sensorial & & & & \\
\hline Excelente & 1 gr & $2 g r$ & $3 g r$ & $4 g r$ \\
Bueno & 0 & 1 & 0 & 1 \\
Regular & 5 & 5 & 5 & 1 \\
Malo & 6 & 5 & 5 & 3 \\
& 0 & 0 & 1 & 6 \\
\hline
\end{tabular}

Hp:La calidad sensorial final del producto y el Pcontenido del "cchacco" son variables indepen dientes.

$$
\tilde{o}_{i j}=\left(\widetilde{O}_{i}\right) \cdot\left(\widetilde{O}_{j} j\right) \quad \begin{aligned}
& j->1, \ldots 4 \\
& j->1, . .
\end{aligned}
$$

Ha: La calidad sensorial y el contenido de cchacco no son variables independientes, al menos de un ${ }_{\mathrm{ij}}$ es diferente del respectivo (ठі.)(ठ.j).

Prueba estadística : $X^{2} 9 \mathrm{gl}$

Para un nivel de significación del $5 \%$ y $2,5 \%$ :
a) Se acepta $\mathrm{Hp}$ si $Q^{\text {cal }} \quad 16,9$
Se rechaza $H p$ si $Q^{\text {cal }}>16,9$
b) Se acepta Hp si $Q^{\text {cal }} 19,0$
Se rechaza $H p$ si $Q^{\text {cal }}>19,0$
Como $Q^{\text {cal }}=19,819$

Para un nivel de significación de $5 \%$ y $2,5 \%$ se halló evidencia estadística de que la calidad sensorial del producto final no es independiente de la cantidad de "cchacco" presente en las fórmulas.

Es importante indicar que tanto esta evaluación como las siguientes, se aplicaron a personas que rechazaron la forma usual de consumo de "cchacco". Con respecto a sus respuestas, las cuatro formulaciones en base a "cchacco" fueron consideradas excelentes o buenas en un $40,92 \%$ y aprobatorias (excelente-buena-regular) en un $84,10 \%$, tal como se muestra en el siguiente gráfico (Ver Anexo Nro. 01).

Preguntado el panel sobre la asociación que podian

\begin{tabular}{|c|c|}
\hline Sabor Asociado & Nivel de Asociación \\
\hline $\begin{array}{l}\text { A galleta, pan, harina, dulce } \\
\text { A huacatay, paico, pimienta } \\
\text { A maní } \\
\text { A tierra, arcilla } \\
\text { A leche }\end{array}$ & 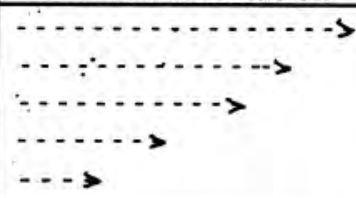 \\
\hline
\end{tabular}
hacer respecto al sabor de las salsas evaluadas se obtuvo las siguientes respuestas:

También se observó la influencia de la temperatura del agua usada para la preparación de las formulaciones, la consistencia que adquieren y el grado de desparramamiento que presentan, expresado en centímetros. La evaluación se hizo para las muestras "L" y " $V$ " (con 1 y 2 gramos de "cchacco") a temperaturas de $23^{\circ} \mathrm{C}$ y $39^{\circ} \mathrm{C}$, tal como puede apreciarse en el gráfico (Ver Anexo Nro. 02).

De los resultados podemos indicar que se aprecia en la salsa una consistencia más fluida, cuando se usa agua a $23^{\circ} \mathrm{C}$, y no así, a la temperatura de $39^{\circ} \mathrm{C}$ en la que, prácticamente, no fluye.

También se hizo evidente que para ambas temperaturas de trabajo, la muestra "L», que posee menor cantidad de "cchacco", presenta un mayor 
grado de desparramamiento $\left(12,9 \mathrm{~cm}\right.$ a $23^{\circ} \mathrm{C}$, y $4,7 \mathrm{~cm}$ a $39^{\circ} \mathrm{C}$ ), en comparación con la muestra " $\mathrm{V}$ " (con $11,3 \mathrm{cms}$ a $23^{\circ} \mathrm{C}$, y $3,6 \mathrm{~cm}$ a $39^{\circ} \mathrm{C}$ ). De estos valores podemos deducir que un incremento de $16^{\circ} \mathrm{C}$ en la temperatura del agua, redujo el grado de desparramamiento de la muestra " $L$ " en un $63,56 \%$ y de la muestra " $V$ " en un $68,14 \%$.

Por otro lado, a $23^{\circ} \mathrm{C}$, se necesitó 19 minutos para que el grado de desparramamiento de la muestra " $L$ " se estabilizara, consiguiéndose este mismo efecto en la muestra " $\mathrm{V}$ " en los diez minutos iniciales; es decir, casi en la mitad del tiempo, al duplicarse la cantidad de "cchacco". Este comportamiento no fue similar a la temperatura de $39^{\circ} \mathrm{C}$, para la cual las gráficas son casi horizontales, debiéndose todo ello, sin duda, a las propiedades ecológicas de la arcilla.

Seguidamente, y con la finalidad de seleccionar la mejor prueba, se aplicó el test de Ranking mediante la evaluación de los atributos: color, olor, sabor, consistencia y aspecto general en presencia de un estandar. Los resultados fueron los siguientes:

a) Para el atributo color:

\begin{tabular}{|l|cccccc|}
\hline Panel & Patrón & \multicolumn{4}{|c|}{ Tratamientos } \\
\hline TOTAL 11 & 21 & & 24 & 34 & 41 & 45 \\
\hline
\end{tabular}

A un nivel de significación del 5\% (21-34) no existe diferencia entre el estándar y las muestras "L» $y$ " $V$ ", habiendo sido rechazadas las caracteristicas de color que presentan las fórmulas " $\mathrm{B}$ " $\mathrm{y}$ " $\mathrm{K}$ ".

b) Para el atributo olor:

De acuerdo al nivel de significación ya establecido y con los siguientes datos :

\begin{tabular}{|cc|ccccc|}
\hline \multicolumn{2}{|c|}{ Panel } & Patrón & \multicolumn{4}{c|}{ Tratamientos } \\
& & $L$ & V & B & $K$ \\
\hline TOTAL & 11 & 24 & 25 & 29 & 37 & 50 \\
\hline
\end{tabular}

no se halló diferencia entre la muestra patrón con los tratamientos "L» $\mathrm{y}$ " $\mathrm{V}$ ", pero sí rechazo de las fórmulas « $B$ " $y$ «K», respecto al estándar.

c) Para el atributo sabor:

\begin{tabular}{|c|cccccc|}
\hline \multirow{2}{*}{ Panel } & \multirow{2}{*}{ Patrón } & \multicolumn{4}{|c|}{ Tratamientos } \\
& & \multicolumn{2}{c}{ L } & V & B & K \\
\hline TOTAL 11 & 36 & 20 & 21 & 37 & 50 \\
\hline
\end{tabular}

La única muestra aceptada, respecto al par (21-34) para $5 \%$ de significación, fue la denominada « $L$ ", mientras que tanto el patrón como las formulaciones «B" $\mathrm{y}$ "K», fueron significativamente diferentes a la elegida como la mejor, con un $95 \%$ de seguridad que lo objetable de las muestras no se debe al azar.

d) Para el atributo consistencia:

Los valores de este cuadro :

\begin{tabular}{|ll|llllll|}
\hline Panel & Patrón & \multicolumn{4}{|c|}{ Tratamiento } \\
& & & & L & V & B & K \\
\hline TOTAL & 11 & 16 & 20 & 40 & 41 & 48 \\
\hline
\end{tabular}

indican de manera análoga a las anteriores, que para ésta evaluación se tuvo como mejor prueba, la empleada como patrón seguida de la fórmula " $L$ ", no así en los otros casos ("V», "B" y "K») para la que el panel considera diferencia significativa de calidad y las catalogó como inaceptables.

\section{e) Para el aspecto general:}

Las respuestas obtenidas del panel evaluador fueron las siguientes :

\begin{tabular}{|cc|cccccc|}
\hline Panel & Patrón & \multicolumn{4}{|c|}{ Tratamiento } \\
& & & L & V & B & K \\
\hline TOTAL & 11 & 16 & & 20 & 36 & 41 & 51 \\
\hline
\end{tabular}

El mejor aspecto general correspondió al patrón seguido de la muestra " $L$," mientras que para las otras formulaciones se detectó rechazo por parte del panel evaluador.

Es importante indicar que el sabor de las diferentessalsas fue asociado con productos como pimienta, mani, grasa, arena o un sabor ligeramente astringente.

Finalmente se probó tres alternativas de sabor en base a la fórmula codificada con la letra "L».

Los resultados obtenidos de la prueba de Ranking para tres tratamientos con el par (18-26) a un nivel de significación de $5 \%$ fueron los siguientes :

\begin{tabular}{|c|cccc|}
\hline Panel & Patrón & \multicolumn{3}{|c|}{ Tratamientos } \\
& & $\mathrm{M}$ & $\mathrm{E}$ & $\mathrm{X}$ \\
\hline TOTAL 11 & 20 & 17 & 26 & 27 \\
\hline
\end{tabular}

En este caso, se consideró el mejor sabor al presentado por la fórmula identificada con la letra " $\mathrm{M}$ ». 
El patrón no mostró diferencia significativa con la muestra «E» que contenía nueces. Por el contrario, los evaluadores rechazaron la codificada con la letra " $X$ " que ocupó el último lugar respecto al estándar empleado.

Tanto la muestra "L" como la « $\mathrm{M}$ ", fueron analizadas para determinar su composición proximal final con los siguientes resultados:

CUADRO NRO. 5: COMPOSICION PROXIMAL DE LAS SALSAS DE CHACCO CON Y SIN MANI

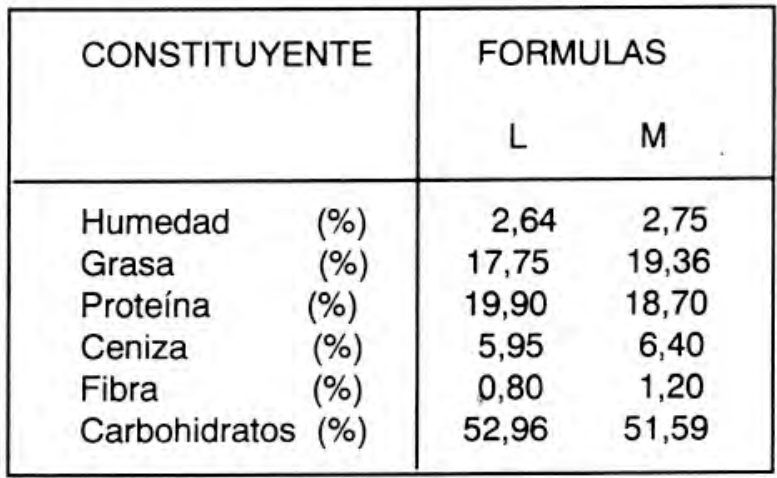

Así podemos observar que la humedad de 2,64\% para la fórmula " $L$ " y de $2,75 \%$ para la fórmula " $M$ ", son bastante bajas y muy cercanas a las que suelen poseer productos deshidratados en polvo, como por ejemplo leche instantaneizada (con 2,8\% de humedad).

El contenido de grasa fue de $17,75 \%$ y de $19,46 \%$ en los productos. Porcentajes que si bien son altos, comparables con la cantidad de grasa que se halla presente en la carne de cerdo $(15,1 \%)$ o frejol soya $(18,9 \%)$, indican que la incorporación de maní (con $48,2 \%$ de grasa) en la formulación «M», representó apenas, un incremento de $1,61 \%$ en este constituyente analizado.

Por otro lado, el porcentaje de proteínas consegido en las formulaciones $(19,90 \%$ y $18,70 \%)$, es semejante a las determinadas en carnes de mamíferos y aves, o en cereales altamente proteicos como la cañihua (con $17,6 \%$ ). Este aporte es fundamental y procede esencialmente de la proteína láctea constituída principalmente por caseína, cuya calidad y características son ampliamente conocidas. Para ambos casos, es importante notar que se consiguió elevar considerablemente el porcentaje de este macro nutriente ya que para el "cchacco" puro es de sólo $0,0174 \%$.

Respecto al contenido de ceniza determinado para ambas muestras, es significativo el aporte mineral del "cchacco". Así tenemos que en la fórmula "L" fue de
$5,95 \%$ y $6,40 \%$ en la fórmula " $\mathrm{M}$ "; semejantes a los hallados en jamón fresco y crudo $(5,4 \%)$ y galletas «pret zels" (5,5\%). Finalmente, al evaluar el contenido de fibra, se obtuvo $0,8 \%$ para la salsa denominada " $L$ " $y$ $1,2 \%$ para la salsa codificada como «M". Valores muy similares a los representados para castañas frescas 0 algunos tipos de nueces $(1,3 \%$ y $1,1 \%$ respectivamente). Mientras que el contenido de carbohidratos $(52,96 \%)$ para el experimento "L" $y$ $51,59 \%$ para el " $\mathrm{M}$ », se asemeja al promedio reportado para diferentes tipos de panes y germen de trigo.

Estos valores indican que las formulaciones preparadas, poseen porcentajes altos en los constituyentes evaluados, especialmente proteína, grasa, cenizas y carbohidratos, además de la presencia de fibra.

\section{CONCLUSIONES}

1). $E l$ «cchacco» es un silicato de alúmina en estado coloidal con las siguientes características físicas y químicas: humedad $7,78 \%$; sólidos totales $92,22 \%$; cenizas $91,80 \%$; nitrógeno $0,0028 \%$, $\mathrm{pH}$ de 6 ; densidad de carga de $0,6936 \mathrm{gr} / \mathrm{ml}$; glucosa $225 \mathrm{mg} \%$; y 3,58 $\mathrm{mg} \%$ de creatinina.

2). El análisis microbiológico del»cchacco" ha demostrado que su consumo, pese a las condiciones de manipuleo, no puede producir enfermedades de etiología microbiana; por lo tanto su calidad sanitaria es óptima y estable, no necesitando inclusive de la adición de conservadores.

3). Hecha la formulación en forma de salsa, buscando la aplicación tecnológica para consumo de "cchacco", se determinó, aplicando un test sensorial descriptivo y considerando un nivel de significación de $5 \%$ y $2,5 \%$ de evidencia estadística, que la calidad sensorial de las formulaciones no es independiente de la cantidad de "cchacco" añadido.

4). Mediante la prueba de Ranking se evaluó lasdiferentes formulaciones (4, con un estándar) en relación a los atributos color, olor, sabor, consistencia y aspecto general; determinándose que la formulación más aceptada, respecto al patrón es aquella que contiene 1 gramo de «cchacco» (muestra "L»).

5).Para incidir en el atributo sabor se aplicó nueva mente el método de Ranking, consiguiendo que la fórmula codificada con la letra «M» (4,5 gr. de maní) tenga mayor aceptación. 
6).El análisis proximal de las muestras "L» $\mathrm{y}$ "M» revelan porcentajes altos de constituyentes básicos

\section{REFERENCIAS BIBLIOGRAFICAS}

(1) En Vocabulario de la lengua aymara.

(2) En La Nutrición en el Antiguo Perú; Edit. Bco. Central de Reserva, Perú, 1988.

(3) Véase Sigurd E: Manual de Nutrición; Ed. Continental, Méjico, 1982.

(4) BUSS D., TYLER H., BARBER S. y CRALY H: Manual de Nutrición, Ed. Acrílica. España. s/f.

(5) FRISANCHO I: Historia del Desarrollo Científico y Tecnológico del Alt. Peruano; 1990.

(6) MUÑOZ A.: Alimentación y Nutrición; Ed. Agra ria y CONCYTEC, Lima, 1990.

(7) MOSSEL D. Y QUEVEDO F.: Microbiológica de los Alimentos; España, 1982.

(8) JUL UVERLY : Evaluación de la Calidad; Dina marca s/f.

(9) AQUINO R.: Métodos para análisis de aguas y residuos sólidos; 1989; y UNALM: Manual de Nutrición, 1976.

(10) BUSS Y OTROS: íbid. (grasa 17,75 y 19,36 ; proteína 19,90 y 18,70 ; ceniza 5,95 y 6,40 respectivamente) como un aporte de los insumos de ambas fórmulas.

(11) GUERRERO ALVA, D.: Elaboración de Queso tipo Roquefort; UNALM, 1987.

(12) UREÑA M.: U Tesis, UNALM, 1987.

(13) COLLAZOS C.: La Composición de los Alimentos Peruanos; Ed. Ministerio de Salud, 3ra. Edic. Lima.

(14) POTTER N.: La Ciencia de los Alimentos; Ed. Edutex, Méjico, 1973.

(15) PEARSON J.: Técnicas de Laboratorio para Análisis de Alimentos; Ed. Acribia, 1986.

(16) MUÑOZ A.; íbid.

(17) DICCIONARIO ENCICLOPEDICO HISPANOAMERICANO; Tms. 6,10 y 16; España.

(18) SIGURD Y OTROS; íbid.

(19) MUÑOZ A.: íbid.

(20) CONFERENCIA SOBRE CULTIVOS ANDINOS, EN SERVI EXPOALIMENTARIA 1994; además, véase Antúnez de Mayolo, Ibid.' '. Ingeniera de Industrias Alimentarias.

\section{CALIFIACION DE LA CALIDAD DE LAS FORMULACIONES EN BASE A CCHACCO}

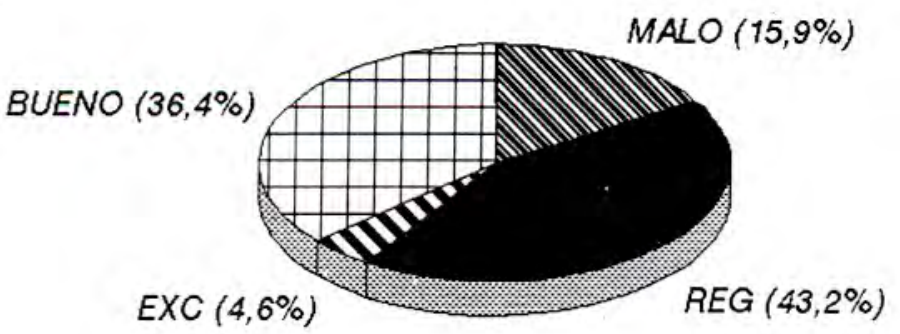



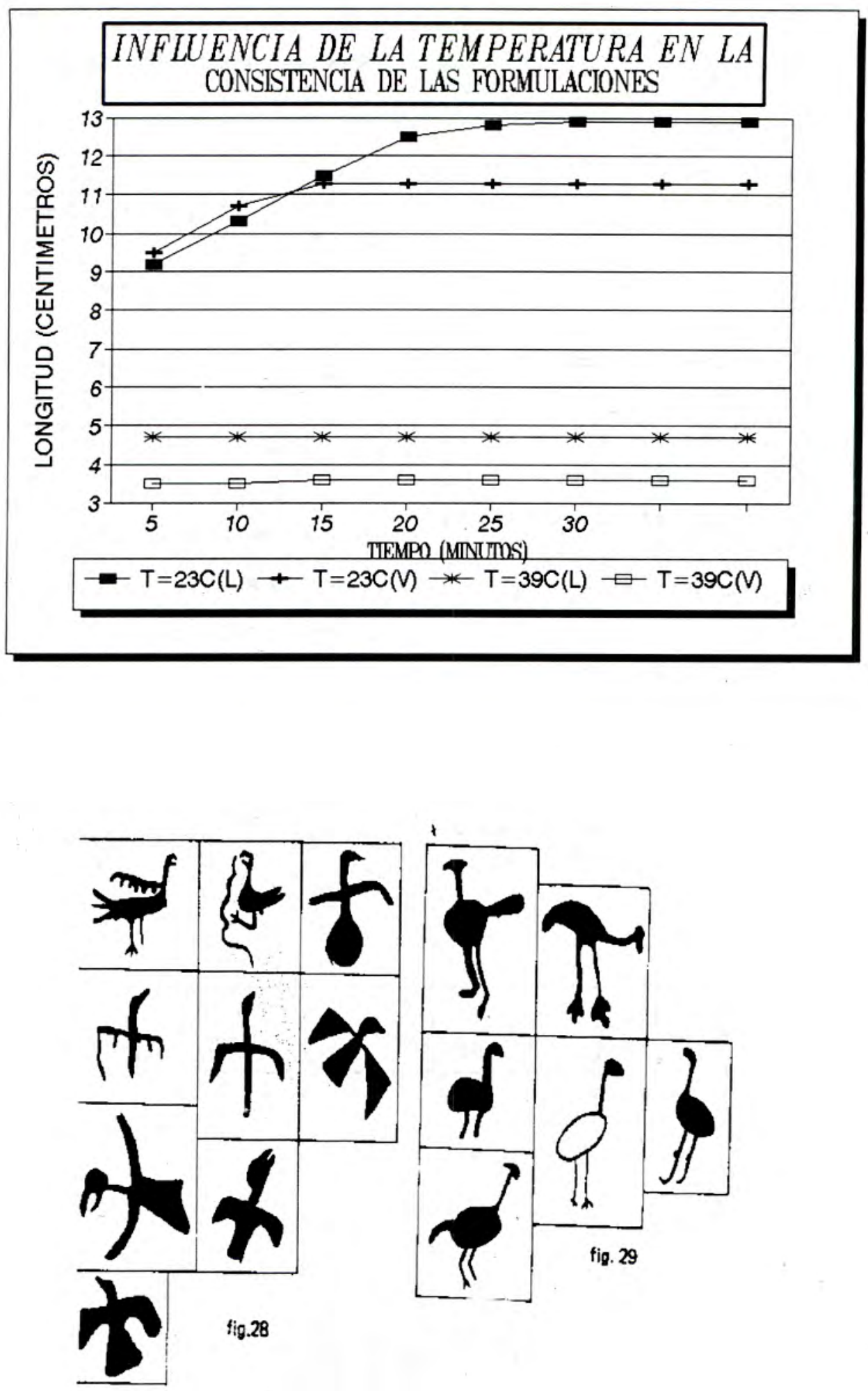